\title{
Técnicas de elicitação de requisitos no desenvolvimento de software: uma revisão sistemática da literatura
}

\section{Requirements elicitation techniques for software development: a systematic review of literature}

\author{
Naiara Crislaine Alflen ${ }^{1}$, Edmir Parada Vasques Prado ${ }^{2}$ \\ ${ }^{1}$ Universidade de São Paulo (USP), São Paulo, SP, Brasil. ORCID: https://orcid.org/0000-0001-9666-1417 \\ 2 Universidade de São Paulo (USP), São Paulo, SP, Brasil. ORCID: http://orcid.org/0000-0002-3505-6122
}

Autor para correspondência/Mail to: Naiara Crislaine Alflen, naiara.alflen@usp.br

Recebido/Submitted: 19 de outubro de 2020; Aceito/Approved: 22 de novembro de 2020

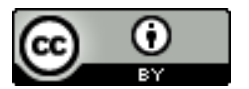

Copyright (C) 2021 Alflen \& Prado. Todo o conteúdo da Revista (incluindo-se instruções, política editorial e modelos) está sob uma licença Creative Commons Atribuição 4.0 Internacional. Ao serem publicados por esta Revista, os artigos são de livre uso em ambientes educacionais, de pesquisa e não comerciais, com atribuição de autoria obrigatória. Mais informações em http://revistas.ufpr.br/atoz/about/submissions\#copyrightNotice.

\begin{abstract}
Resumo
Introdução: A elicitação de requisitos (ER) é uma tarefa difícil na qual é necessário lidar com ambiguidade de informações, dados incompletos e inconsistentes. Ela busca descobrir e entender o real problema e as necessidades dos usuários. O sucesso do desenvolvimento de software depende da elicitação correta dos requisitos, e a qualidade deles é influenciada pelas técnicas usadas. Dentro deste contexto, o objetivo desta pesquisa é identificar as técnicas de ER mais citadas na literatura em projetos de desenvolvimento de software. Método: Foi realizada uma revisão sistemática da literatura, que analisou 61 artigos da base de dados Scopus. Resultados: Foram identificadas as 10 técnicas de ER mais citadas na literatura. A classificação dessas técnicas mostrou que aquelas obtidas pelo envolvimento de grupos de stakeholders foram as menos citadas. Conclusão: A pesquisa identificou a oportunidade e relevância para o desenvolvimento de pesquisa acadêmica descritiva ou confirmatória sobre técnicas de ER baseadas na interação entre grupos de usuários e membros da equipe de desenvolvimento de software.
\end{abstract}

Palavras-chave: Elicitação de requisitos; Sistemas de informação; Participação da equipe.

\begin{abstract}
Introduction: Requirements elicitation (RE) is a difficult task in which there are issues related to information ambiguity, incomplete and inconsistent data. It seeks to discover and understand the users' problems and needs. The success of software development depends on the correct elicitation of requirements, and its quality is influenced by the techniques used. This research aims to identify the RE techniques most cited in the literature of software development projects within this context. Method: a systematic literature review was carried out, which analyzed 61 articles from the Scopus database. Results: We identified the 10 RE techniques most cited in the literature. The ranking of these techniques showed that those obtained by stakeholders' groups' involvement were the least mentioned. Conclusions: the research identified the opportunity and relevance for developing a descriptive or confirmatory analysis of RE techniques based on the interaction between groups of users and members of the software development team.
\end{abstract}

Keywords: Requirements elicitation; Information systems; Team participation.

\section{INTRODUÇÃO}

A engenharia de requisitos é a fase mais complexa no desenvolvimento de software (Buitrón, Flores-Rios, \& Pino, 2018; Fernandes et al., 2012; Kiran \& Ali, 2018). Segundo Alexa e Avasilcai (2018), uma das etapas da engenharia de requisitos é a elicitação de requisitos (ER), que é dedicada à descoberta, extração e revelação das necessidades dos usuários.

A ER é o entendimento da real necessidade do usuário (Hickey \& Davis, 2003). Ela é uma tarefa difícil na qual é necessário lidar com ambiguidade de informações, dados incompletos e inconsistentes, em que os requisitos não são claramente conhecidos (Vijayan, Raju, \& Joseph, 2016). Além disso, a ER não se refere apenas a escrever requisitos, mas em descobrir e entender o real problema e as necessidades dos usuários (Araujo, Anjos, \& Silva, 2015). A incompreensão da necessidade do usuário é um dos principais fatores de fracasso de um projeto (Gonzales \& Leroy, 2011). Na maioria das vezes, os usuários têm dificuldade em expressar seus requisitos (Nuseibeh \& Easterbrook, 2000). Segundo Mishra, Mishra, e Yazici (2008), a maior falha em projetos de software são requisitos incompletos e incorretos.

Metodologias convencionais para ER se concentram em reunir todos os requisitos do software para documentá-los e posteriormente passam para a fase de desenvolvimento (Alexa \& Avasilcai, 2018; Batool et al., 2013). Porém, em ambientes em constante evolução, essas metodologias não são abordagens adequadas, pois no cenário atual a mudança é considerada uma característica essencial do desenvolvimento de software (Jayatilleke \& Lai, 2018). Nesse contexto, Knauss, Yussuf, Blincoe, Damian, e Knauss (2018) consideram a engenharia de requisitos tradicional insuficiente para obter uma ER adequada. De forma semelhante, Batool et al. (2013) e Sheffield e Lemétayer (2013) mencionam a necessidade de um processo de desenvolvimento de software flexível e rápido, em que é necessário trabalhar com ciclos curtos de entregas para lidar com as mudanças e incertezas de requisitos. Como consequência, as metodologias ágeis com entregas rápidas e incrementais suportam mudanças de requisitos 
e o processo de elicitação ocorre no decorrer do desenvolvimento do software (Asghar, Tabassum, Bhatti, \& Jadi, 2017).

A qualidade dos requisitos é influenciada pelas técnicas utilizadas na ER. Isso ocorre, segundo Hickey e Davis (2003), pois a ER é um momento de aprendizado e comunicação com os usuários. Porém, existem técnicas para ER nas quais a participação do usuário não é abordada, e há outras técnicas nas quais a participação dele é fundamental. Independentemente da maior ou menor participação do usuário, Babar, Bunker, e Gill (2018) alegam que o sucesso do desenvolvimento de software depende da elicitação correta dos requisitos. Dentro deste contexto, o objetivo desta pesquisa é identificar as técnicas de ER mais citadas na literatura em projetos de desenvolvimento de software.

\section{MÉTODO DE PESQUISA}

A pesquisa proposta neste trabalho caracteriza-se por ser um estudo exploratório. Segundo Cervo e Bervian (2007), a pesquisa exploratória visa oferecer informações sobre o objeto de estudo. Dessa forma, esta pesquisa explora as bases de dados científicas para identificar técnicas de ER em projetos de desenvolvimento de software. Este estudo se trata de uma pesquisa qualitativa e transversal do tipo único, pois a coleta de dados foi feita uma única vez. A seguir são apresentadas as etapas da pesquisa e os procedimentos metodológicos para coleta e análise de dados.

\section{Etapas da Pesquisa}

As etapas da pesquisa estão esquematizadas na Figura 1 serviram como base para realização desta pesquisa. Inicialmente, a partir de uma revisão sistemática da literatura (RSL), foram identificadas e listadas as técnicas utilizadas para ER em projetos de desenvolvimento de software. Em seguida, a partir dos artigos selecionados, as técnicas foram analisadas, identificando suas características e frequência de uso no ambiente organizacional.

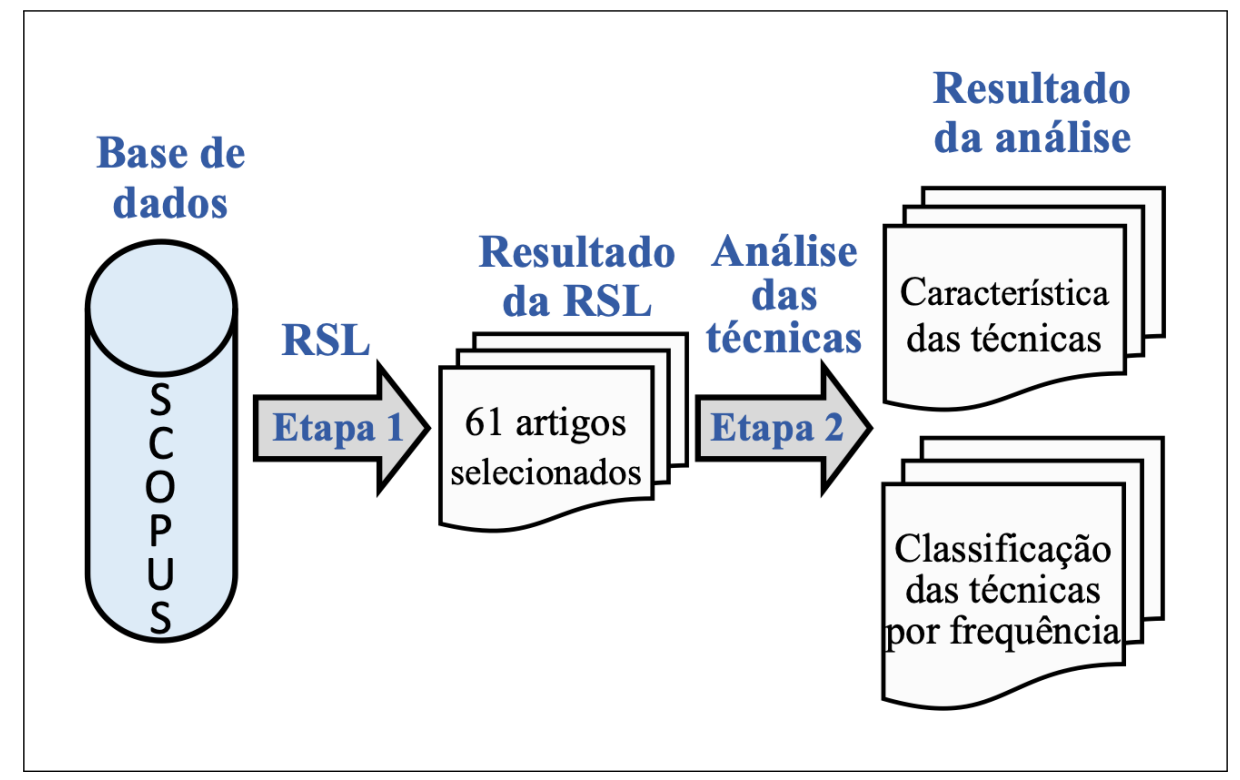

Figura 1. Etapas da pesquisa.

\section{Coleta e Análise dos Dados}

A identificação de técnicas de ER foi realizada por meio de uma RSL. A RSL é um método para identificar e analisar trabalhos disponíveis nas bases de dados científicas e responder a questões de pesquisa (Baptista \& de Campos, 2007). Kitchenham (2004) menciona que a RSL usa um método rigoroso, confiável e auditável. Para realizar a RSL, foi utilizado o protocolo proposto por Kitchenham e Charters (2007). Esse protocolo estabelece estratégias de pesquisa para a estruturação do trabalho e para identificação e avaliação dos materiais encontrados. O protocolo foi realizado em três fases: planejamento, seleção e resultados.

\section{Planejamento}

O planejamento da RSL é constituído por dois itens: definição do objetivo e definição do protocolo de pesquisa. O objetivo desta RSL é identificar técnicas de ER, e a definição do protocolo foi realizada em três etapas apresentadas a seguir:

a) Questão de pesquisa. A ER é um tema abordado em diferentes áreas de conhecimento e nesta pesquisa foi especificada a área de desenvolvimento de software. Assim, a questão definida para esta RSL foi: Quais são as técnicas de ER utilizadas em projetos de desenvolvimento de software? 
b) Identificação dos estudos. Foi realizada uma busca ampla nas bases de dados por estudos que respondam à questão de pesquisa proposta. Esta RSL utilizou a base de dados Scopus (www.scopus.com), pois essa base indexa os trabalhos das bibliotecas ACM Digital Library e IEEEXplore. A definição das palavras chaves considerou os termos "elicitação de requisitos", "técnicas" e "software", na língua inglesa e com seus respectivos sinônimos, conforme apresentado no Quadro 1.

c) Critérios de seleção dos estudos. Adotaram-se critérios de inclusão, exclusão e qualidade para a seleção dos artigos. Os trabalhos foram incluídos quando atenderam aos três critérios de inclusão, mas eliminados ao satisfazer um dos critérios de exclusão. Os critérios definidos foram:

- Critérios de inclusão: (I1) artigo que apresentem técnicas para ER; (I2) artigos que apresentam técnicas para ER no desenvolvimento de software; e (I3) artigos publicados no período de 2009 a 2020.

- Critérios de exclusão: (E1) artigo não é apresentado na sua totalidade em inglês; (E2) artigo não está relacionado as áreas Ciências da Computação, Sistemas de Informação ou Engenharia; (E3) artigo não é de periódico ou conferência revisada por pares; (E4) artigo é de workshop, lecture notes, work in progress ou artigo curto; (E5) artigo não está disponível eletronicamente ou com restrições de acesso; e (E6) artigo não é um estudo primário.

- Critério de qualidade: (C1) baseado na pontuação média de citações de periódicos da base de dados Scopus.

\begin{tabular}{|l|l|}
\hline Palavra chave & Sinônimos \\
\hline Requirements elicitation & requirements gathering \\
\hline Techniques & methods OR procedures OR tools OR artifacts OR specification \\
\hline Software & system OR systems \\
\hline \multicolumn{1}{|c|}{ Termo de busca } \\
\hline $\begin{array}{l}\text { TITLE-ABS-KEY (("Requirements elicitation" OR "requirements gathering") AND (Techniques OR methods OR } \\
\text { procedures OR tools OR artifacts or specification) AND (software OR system OR systems)) }\end{array}$ \\
\hline
\end{tabular}

Quadro 1. Estratégia de busca aplicada na base de dados

Foram selecionados os artigos pertencentes ao quartil com maior pontuação.

\section{Seleção de Artigos}

A busca na base foi realizada no mês de maio de 2020. A aplicação do termo de busca na base de dados Scopus resultou em 1.972 artigos. A partir desses artigos, foram aplicados os critérios de inclusão, exclusão e qualidade: critérios de inclusão I1, I2 e I3, resultaram em 972 artigos; critério de exclusão E1, E2, E3, E4, E5 e E6 resultaram em 197 artigos; e critério de qualidade C1 resultou em 61 artigos.

\section{APRESENTAÇÃO E ANÁLISE DOS RESULTADOS}

As técnicas de ER encontradas na literatura, por meio da RSL, são de trabalhos relacionados ao processo de desenvolvimento de software. No entanto, essas mesmas técnicas também são encontradas em trabalhos de outros campos de conhecimento. Em grande parte dos trabalhos, foram encontradas mais de uma técnica no processo de ER. Entretanto, foram encontradas diversas nomenclaturas para técnicas similares como, por exemplo, as técnicas de os termos "questionary" e "survey".

As técnicas similares foram agrupadas e identificaram-se 40 técnicas para ER. Em alguns trabalhos, as técnicas são descritas e em outros apenas citadas. Na Tabela 1 são apresentadas as técnicas em ordem decrescente de frequência de citação e os artigos nos quais elas foram encontradas. Sete técnicas apresentaram somente uma citação e não estão incluídas na tabela. 


\begin{tabular}{|c|c|}
\hline Técnica & Artigos relacionados no apêndice $A$ \\
\hline Entrevistas & $\begin{array}{l}{[02][04][05][06][07][09][10][11][12][14][15][17][18][20][21][22][24]} \\
{[25][28][29][30][31][32][33][34][36][38][40][41][43][44][45][46][47]} \\
{[48][52][53][54][55][56][57][58][59][60][61]}\end{array}$ \\
\hline Questionário & $\begin{array}{l}{[03][04][05][07][08][09][10][13][14][15][18][20][22][24][26][28][29]} \\
{[30][34][35][36][37][41][43][44][45][46][47][48][50][55][56][58][59]}\end{array}$ \\
\hline Protótipo & $\begin{array}{l}{[02][03][05][07][08][09][10][12][13][15][17][21][22][27][31][33][34]} \\
{[39][41][43][47][48][53][54][59]}\end{array}$ \\
\hline Casos de uso & $\begin{array}{l}{[01][05][07][09][10][14][15][16][19][22][26][32][35][36][38][42][43]} \\
{[45][47][49][50][56][57][60][61]}\end{array}$ \\
\hline Brainstorming & $\begin{array}{l}{[05][07][14][15][16][17][27][28][31][33][34][36][37][39][40][41][44]} \\
{[45][46][47][54][55][58][59]}\end{array}$ \\
\hline Cenário & $\begin{array}{l}{[01][02][07][08][11][14][16][18][21][22][27][28][33][34][35][38][41]} \\
{[47][49][50][51][54][61]}\end{array}$ \\
\hline Feedback & $\begin{array}{l}{[01][03][04][07][08][11][12][21][24][25][27][33][39][40][44][47][50]} \\
{[52][55]}\end{array}$ \\
\hline Workshop & {$[04][05][11][12][14][15][16][28][32][34][38][41][44][46][48][50][54]$} \\
\hline Grupo focal & {$[03][07][14][15][17][21][22][24][31][38][39][41][46][48]$} \\
\hline História de usuário & {$[07][11][14][17][19][22][23][30][34][48][49][54]$} \\
\hline Observação & {$[02][05][07][11][17][21][22][27][30][31][38]$} \\
\hline Etnografia & {$[04][22][23][24][38][40][42][43][45][61]$} \\
\hline JAD / RAD & {$[04][07][28][38][41][42][44][50][52][55]$} \\
\hline Storyboards & {$[15][21][23][36][38][39][45]$} \\
\hline Análise de documento & {$[14][15][25][38][41][52][60]$} \\
\hline Linguagem natural & [11][12][15][17][20][31] \\
\hline Storytelling & [08] [22] [23] [39] [42] [45] \\
\hline Goal Based & [01][29][34][38][51] \\
\hline Personas & {$[08][24][41][53][60]$} \\
\hline Crowdsourcing & [12] [16] [19][20] [60] \\
\hline Quality Function Deployment & {$[29][34][57][60]$} \\
\hline Análise de Domínio & [34][49][57][60] \\
\hline Ontologia & {$[18][38][57][60]$} \\
\hline $\begin{array}{l}\text { Issue-based } \\
\text { systems }\end{array}$ & {$[45][55][57][60]$} \\
\hline Win-Win & [44] [54] [55] \\
\hline Repertory grids & {$[18][25][41]$} \\
\hline $\begin{array}{l}\text { Controlled requirements expres- } \\
\text { sion }\end{array}$ & [57][60] \\
\hline Critical discourse analysis & [57][60] \\
\hline Ferramentas colaborativas & [49][59] \\
\hline Introspection & [15][34] \\
\hline Appreciative Inquiry & [31] [47] \\
\hline Experiência do usuário & {$[08][22]$} \\
\hline Gamificação & [39][42] \\
\hline
\end{tabular}

Tabela 1. Artigos selecionados na RSL

A análise das técnicas de ER está apresentada em dois tópicos. No primeiro são apresentadas as características e frequências de uso das técnicas. No segundo as técnicas são descritas e analisadas, ou seja, as técnicas são classificadas em relação às suas fontes de obtenção dos requisitos e são apontados os benefícios e limitações das técnicas mais citadas na literatura.

\section{Características e Frequência de Uso das Técnicas}

Aplicou-se a estatística quartil à frequência das técnicas de ER encontradas na literatura. O primeiro quartil foi considerado, para efeito de análise nesta pesquisa, como contendo as técnicas de ER mais citadas na literatura, ou seja, o conjunto que contém $25 \%$ das técnicas mais citadas. Esse conjunto é composto pelas 10 técnicas, que estão apresentadas a seguir:

a) Entrevista. Foi a técnica mais mencionada nos artigos. Ela esteve presente em 45 dos 61 artigos selecionados. A entrevista geralmente envolve um representante da equipe do projeto com um stakeholder e é conhecida por ser a mais comum das técnicas de ER.

b) Questionário. Foi a segunda técnica mais citada, encontrada em 34 dos 61 artigos. Do ponto de vista de 
Abd-Elmonem, Nasr, e Gheith (2017), o questionário é uma técnica tradicional de ER e habitualmente utilizada. É uma ferramenta simples, geralmente aplicada na fase inicial da ER para coletar o máximo de requisitos de diferentes stakeholders que podem estar em lugares distintos.

c) Prototipação. É uma versão incompleta ou inicial do software. Ou seja, ela pode ser descartável ou evolutiva (Younas, Jawawi, Ghani, \& Kazmi, 2017). Ela coleta o feedback dos stakeholders e identifica alterações que devem ser incorporadas na próxima versão.

d) Caso de uso. Representa um possível uso do sistema por um ator usando algum serviço. O caso de uso narra a interação entre o sistema e os atores envolvidos. Para Hajri, Goknil, Briand, e Stephany (2018) é a principal técnica empregada para extrair requisitos e se comunicar com clientes.

e) Brainstorming. É uma reunião na qual cada participante pode expressar livremente os requisitos do sistema. Para Younas et al. (2017), é uma maneira de sintonizar a mente do usuário em relação aos requisitos.

f) Cenários. Representam as interações dos usuários com o sistema. Permitem descrições do processo atual e futuro, necessário para o desenvolvimento do projeto de software. Segundo Adem e Kasirun (2010), eles auxiliam a descobrir as metas do software e contemplam a interação com o usuário.

g) Feedback. Segundo Hosseini et al. (2015), esta técnica propicia aos participantes a sensação de que suas ideias são importantes e pode levar à convergência de opiniões.

h) Workshop. É uma técnica colaborativa para definir os requisitos de um software e pode ser utilizado para esclarecer ambiguidades (Angelis, Ferrari, Gnesi, \& Polini, 2018; Mishra, Aydin, Mishra, \& Ostrovska, 2018). Ele é uma espécie de seminário ou grupo de discussão em que um palestrante apresenta um conteúdo específico e incentiva a reflexão no grupo.

i) Grupo focal. É uma discussão objetiva que introduz um tópico a um grupo de participantes e direciona sua discussão sobre o tema, de uma maneira não-estruturada. Para Younas et al. (2017), é uma maneira de conhecer as vontades e percepções do usuário em relação ao software, assim como a definição dos requisitos.

j) Histórias de usuários. São breves descrições das funcionalidades do software (Mobasher \& ClelandHuang, 2011; Younas et al., 2017) e são discutidas durante todas as fases do projeto para esclarecimento dos requisitos (Knauss et al., 2018; Younas et al., 2017).

\section{Descrição e Análise das Técnicas}

Classificação em Relação à Fonte de Obtenção dos Requisitos

Batista (2003) classificou as técnicas de ER em cinco categorias segundo as fontes de obtenção dos requisitos. As técnicas de ER identificadas nesta pesquisa foram classificadas com base nessas categorias e estão apresentadas na Tabela 2.

\begin{tabular}{|c|c|c|c|}
\hline Fonte dos requisitos & Técnicas & Ordem de citação RSL & Classificação média \\
\hline \multirow{2}{*}{ Individual } & Questionário & 2 & \multirow{2}{*}{4,5} \\
\hline & Feedback & 7 & \\
\hline \multirow{3}{*}{ Grupo } & Brainstorming & 5 & \multirow{3}{*}{7,3} \\
\hline & Workshop & 8 & \\
\hline & Grupo focal & 9 & \\
\hline \multirow{5}{*}{ Mista } & Entrevista & 1 & \multirow{5}{*}{4,8} \\
\hline & Prototipagem & 3 & \\
\hline & Casos de Uso & 4 & \\
\hline & Cenários & 6 & \\
\hline & História de usuário & 10 & \\
\hline
\end{tabular}

Tabela 2. Técnicas mais citadas de ER e classificadas pela fonte dos requisitos

Nenhuma das dez técnicas mais citadas na RSL tem a documentação ou a observação como fonte de requisitos. Elas foram classificadas em apenas três fontes: individual, grupo e mista. Isso indica que as técnicas mais citadas são baseadas na interação entre usuário e desenvolvedor. Por outro lado, as técnicas baseadas em interações individuais ou mistas são mais citadas que as baseadas exclusivamente em grupos, ou seja, as técnicas Brainstorming, Workshop e Grupo focal tiveram uma classificação média de 7,3, inferior aos outros tipos de fontes de requisitos. Por outro lado, há um aumento no uso de técnicas de ER que envolvem mais o usuário e buscam propiciar criatividade e inovação ao processo, assim como o Design Thinking (Araujo et al., 2015).

\section{Benefícios}


Os benefícios obtidos por meio da aplicação dessas dez técnicas atendem as principais questões envolvendo a ER. A técnica de questionário atende à característica de baixo custo. De forma semelhante, segundo Ramakrishnan et al. (2014), a técnica de cenários reduz o tempo gasto no desenvolvimento e, portanto, o custo. O questionário, além de ter baixo custo, permite uma coleta massiva de dados. Al-Qudah, Cristea, e Lei (2013) também destacam esta característica para a técnica de Brainstorming, na qual os dados coletados são posteriormente refinados.

Outra característica observada é a criatividade. Caleb-Solly, Dogramadzi, Ellender, Fear, e Heuvel (2014) destacam que o Brainstorming estimula a criatividade. De forma semelhante, Smith, Strauss, e Maher (2010) alegam que a entrevista reúne informações para novos projetos e permite buscar ideias novas, contribuindo para a criatividade. Isso também pode ser obtido com o uso da técnica de grupo focal. Segundo Alvertis, Papaspyros, Koussouris, Mouzakitis, e Askounis (2016), com a aplicação desta técnica é possível coletar opiniões diversas.

A segurança também é uma questão endereçada por essas técnicas. A técnica de Caso de Uso pode prever o comportamento de um usuário mal-intencionado e o uso indevido do software (Odusote, Daramola, \& Adigun, 2018). Além disso, Odusote et al. (2018) aplicaram o Misuse Case e notaram que ele fornece uma abordagem intuitiva para elicitar requisitos que atendem ao critério de confiabilidade. A técnica de história de usuário, segundo Ramesh e Reddy (2016), pois um caso específico, que são as histórias de abuso, e que descrevem histórias no contexto do invasor do software, contribuindo para endereçar questões de segurança.

Cabe destacar ainda que essas técnicas facilitam o engajamento do usuário, esclarecem problemas e melhoram a definição de requisitos. Para Ramakrishnan et al. (2014) e Hidalga, Hardisty, e Jones (2016), a prototipagem facilita o engajamento do usuário e a identificação precoce de problemas. O Workshop e o Feedback também ajudam na identificação de problemas, pois o primeiro esclarece ambiguidades (Ramakrishnan et al., 2014) e o segundo permite que o usuário final comunique problemas, necessidades e opções ao usar um produto de software (Oriol et al., 2018). Por último, a técnica de Cenários ajuda a reduzir requisitos incompreendidos.

\section{Limitações}

Boa parte das limitações dessas técnicas está associada à pessoa que a aplica e ao usuário. Segundo Gill, Zaidi, e Kiani (2014), o sucesso no resultado de uma entrevista depende da capacidade do entrevistador para conduzir a coleta dos requisitos. Além disso, as técnicas cuja fonte de requisitos é obtida por meio de grupo de pessoas também apresentam essas limitações: (1) para Mishra et al. (2018), a experiência do usuário deve ser considerada para o uso correto da técnica de brainstorming, pois usuários habituados no contexto de desenvolvimento de software conseguem expressar melhor suas necessidades; (2) Fernandes et al. (2012) advertem que, por ser uma técnica colaborativa, no workshop os participantes dominantes e tendenciosos podem inibir as opiniões dos demais participantes; (3) para Pitula e Radhakrishnan (2011), na aplicação do Grupo Focal há a desvantagem dos participantes se sentirem desconfortáveis ao declararem opiniões diferentes daquelas levantadas pelo grupo, além dos participantes dominantes e tendenciosos, que fazem com que ideias válidas de outros participantes fiquem inexploradas (Fernandes et al., 2012).

Outra limitação comum a essas técnicas está associada à questão de tempo e recursos. A primeira técnica que apresenta essa desvantagem é a prototipagem. Gill et al. (2014) destacam que apesar das vantagens da prototipagem, esta apresenta a desvantagem de consumir tempo e recursos. A técnica de cenários apresenta problema similar, pois pode exigir várias reuniões e fazer demonstrações de conceitos com cenários pode demorar um tempo considerável.

Por último, cabe destacar que algumas técnicas apresentam limitações quando usadas para determinados fins. Thomas, Bandara, Price, e Nuseibeh (2014) alegam que os questionários não fornecem informações suficientemente ricas sobre decisões dos usuários e como elas são influenciadas pelo contexto emergente em uma situação particular. Além disso, sua aplicação tem apresentado dificuldades no processo de ER. Outras técnicas não se adequam a determinados tipos de requisitos: Sadiq, Ghafir, e Shahid (2009) afirmam que o Brainstorming não é adequado para a ER de segurança, ou seja, não resulta em um conjunto consistente de requisitos de segurança; e a técnica de cenários não é adequada para o levantamento dos requisitos não funcionais de um sistema.

\section{CONSIDERAÇÕES FINAIS}

O objetivo desta pesquisa foi identificar as técnicas de ER mais citadas na literatura em projetos de desenvolvimento de software. Para atingir este objetivo, efetuou-se uma pesquisa qualitativa e exploratória com base em uma RSL. As conclusões deste trabalho estão apresentadas a seguir.

Técnicas obtidas por meio da interação em grupo foram menos citadas que as demais, apesar da crescente ênfase no envolvimento do usuário. Isto mostra que há oportunidade e relevância para a pesquisa acadêmica que busque descrever e analisar técnicas de ER baseadas na interação entre grupos de usuários e membros da equipe de desenvolvimento de software. A Teoria da Atividade (Wertsch, 1981) pode auxiliar na compreensão de como essa relação social influencia a atividade de ER. 
Esta pesquisa é parte de um projeto de estudo de técnicas de ER. A partir dos resultados desta pesquisa, pretende-se elaborar uma comparação com as técnicas de ER mais utilizadas por empresas públicas e privadas no desenvolvimento de software. E em seguida elaborar proposições para pesquisa confirmatória acerca do uso mais adequado de técnicas de ER em contextos específicos.

\section{APÊNDICE A}

O Quadro 2 a seguir apresenta os artigos da RSL em ordem decrescente do ano de publicação.

(continua)

\begin{tabular}{|c|c|c|c|}
\hline \# & Autores & Título & Ano \\
\hline 01 & Ferraris, D., Fernandez GC. & TrUStAPIS: a trust requirements elicitation method for loT & 2020 \\
\hline 02 & Martinez A. & $\begin{array}{l}\text { An Experience Report on the Use of Experience Maps and } \\
\text { Sketches in a Database Course Project }\end{array}$ & 2019 \\
\hline 03 & Rizk N.M. et al. & $\begin{array}{l}\text { Crowdsourcing based requirements elicitation for eLearning } \\
\text { Systems }\end{array}$ & 2019 \\
\hline 04 & Alwadain A., Alshargi M. & $\begin{array}{l}\text { Crowd-generated data mining for continuous requirements } \\
\text { elicitation }\end{array}$ & 2019 \\
\hline 05 & Martins H.F., et al. & $\begin{array}{l}\text { Design thinking: Challenges for software requirements elici- } \\
\text { tation }\end{array}$ & 2019 \\
\hline 06 & Garcia l. et al. & $\begin{array}{l}\text { Experiences of using a game for improving learning in soft- } \\
\text { ware requirements elicitation }\end{array}$ & 2019 \\
\hline 07 & Fatima R. et al. & $\begin{array}{l}\text { Improving software requirements reasoning by novices: A } \\
\text { story-based approach }\end{array}$ & 2019 \\
\hline 08 & Li C. et al. & $\begin{array}{l}\text { Automatically classifying user requests in crowdsourcing } \\
\text { requirements engineering }\end{array}$ & 2018 \\
\hline 09 & Elmonem M.A. et al. & Automating requirements elicitation of cloud based ERPs & 2018 \\
\hline 10 & Hajri l. et al. & Configuring use case models in product families & 2018 \\
\hline 11 & Knauss E. et al. & $\begin{array}{l}\text { Continuous clarification and emergent requirements flow in } \\
\text { open-commercial software ecosystems }\end{array}$ & 2018 \\
\hline 12 & Oriol M. et al. & $\begin{array}{l}\text { FAME: Supporting continuous requirements elicitation by } \\
\text { combining user feedback and monitoring }\end{array}$ & 2018 \\
\hline 13 & Ohashi K. et al. & $\begin{array}{l}\text { Focusing requirements elicitation by using a UX measure- } \\
\text { ment method }\end{array}$ & 2018 \\
\hline 14 & Babar A. et al. & $\begin{array}{l}\text { Investigating the relationship between business analysts' } \\
\text { competency and IS requirements elicitation: A thematic- } \\
\text { analysis approach }\end{array}$ & 2018 \\
\hline 15 & Mishra D. et al. & Knowledge management in requirement elicitation & 2018 \\
\hline 16 & Odusote B. et al. & $\begin{array}{l}\text { Towards an extended misuse case framework for elicitation } \\
\text { of cloud dependability requirements }\end{array}$ & 2018 \\
\hline 17 & Younas, M. et al. & $\begin{array}{l}\text { Non-Functional Requirements Elicitation Guideline for Agile } \\
\text { Methods }\end{array}$ & 2017 \\
\hline 18 & Dey S., Lee S.-W. & $\begin{array}{l}\text { REASSURE: Requirements elicitation for adaptive socio- } \\
\text { technical systems using repertory grid }\end{array}$ & 2017 \\
\hline 19 & $\begin{array}{l}\text { Raja R.M.R., Satyananda } \\
\text { R.C. }\end{array}$ & $\begin{array}{l}\text { A survey on security requirement elicitation methods: Clas- } \\
\text { sification, merits, and demerits }\end{array}$ & 2016 \\
\hline 20 & Elsaid A.H. et al. & Automatic framework for requirement analysis phase & 2016 \\
\hline 21 & de la Hidalga A.N. et al. & $\begin{array}{l}\text { SCRAM-CK: applying a collaborative requirement enginee- } \\
\text { ring process for designing a web-based e-science toolkit }\end{array}$ & 2016 \\
\hline 22 & Alvertis I. et al. & $\begin{array}{l}\text { Using crowdsourced and anonymized Personas in the re- } \\
\text { quirements elicitation and software development phases of } \\
\text { software engineering }\end{array}$ & 2016 \\
\hline 23 & Maiti R.R., Mitropoulos F.J. & $\begin{array}{l}\text { Capturing, eliciting, predicting, and prioritizing (CEPP) non- } \\
\text { functional requirements metadata during the early stages of } \\
\text { agile software development }\end{array}$ & 2015 \\
\hline 24 & Hosseini M. et al. & Configuring crowdsourcing for requirements elicitation & 2015 \\
\hline 25 & Dey S., Lee S.-W. & $\begin{array}{l}\text { From requirements elicitation to variability analysis using } \\
\text { repertory grid: A cognitive approach }\end{array}$ & 2015 \\
\hline 26 & Faßbender S. et al. & $\begin{array}{l}\text { Problem-based security requirements elicitation and refine- } \\
\text { ment with pressure }\end{array}$ & 2015 \\
\hline 27 & Ellis L. et al. & $\begin{array}{l}\text { Thinking out loud and e-health for coordinated care - Les- } \\
\text { sons from user requirements gathering in the } 4 C \text { project }\end{array}$ & 2015 \\
\hline
\end{tabular}


(continua)

\begin{tabular}{|c|c|c|c|}
\hline \# & Autores & Título & Ano \\
\hline 28 & Caleb-Solly P. et al. & $\begin{array}{l}\text { A mixed-method approach to evoke creative and holistic } \\
\text { thinking about robots in a home environment }\end{array}$ & 2014 \\
\hline 29 & Sadiq M., Jain S.K. & $\begin{array}{l}\text { Applying fuzzy preference relation for requirements prioriti- } \\
\text { zation in goal-oriented requirements elicitation process }\end{array}$ & 2014 \\
\hline 30 & Thomas K. et al. & Distilling privacy requirements for mobile applications & 2014 \\
\hline 31 & Gill K.D. et al. & $\begin{array}{l}\text { Eliciting futuristic end-user requirements through contribu- } \\
\text { tory appreciative inquiry }\end{array}$ & 2014 \\
\hline 32 & Wohlrab R. et al. & $\begin{array}{l}\text { Experience of pragmatically combining RE methods for per- } \\
\text { formance requirements in industry }\end{array}$ & 2014 \\
\hline 33 & Ramakrishnan L. et al. & $\begin{array}{l}\text { Experiences with user-centered design for the tiger's work- } \\
\text { flow API }\end{array}$ & 2014 \\
\hline 34 & Kassab M. et al. & $\begin{array}{l}\text { State of practice in requirements engineering: Contempo- } \\
\text { rary data }\end{array}$ & 2014 \\
\hline 35 & Shirogane J. & Support Method to Elicit Accessibility Requirements & 2014 \\
\hline 36 & Katina P.F. et al. & System requirements engineering in complex situations & 2014 \\
\hline 37 & Al-Qudah D.A. et al. & $\begin{array}{l}\text { An exploratory study to design an adaptive hypermedia sys- } \\
\text { tem for online advertisement }\end{array}$ & 2013 \\
\hline 38 & Al Balushi T.H. et al. & $\begin{array}{l}\text { Eliciting and prioritizing quality requirements supported by } \\
\text { ontologies: A case study using the ElicitO framework and } \\
\text { tool }\end{array}$ & 2013 \\
\hline 39 & Rice M., Carmichael A. & $\begin{array}{l}\text { Factors facilitating or impeding older adults' creative con- } \\
\text { tributions in the collaborative design of a novel DTV-based } \\
\text { application }\end{array}$ & 2013 \\
\hline 40 & Zhang W. et al. & $\begin{array}{l}\text { Feature-oriented Stigmergy-based collaborative require- } \\
\text { ments modeling: An exploratory approach for requirements } \\
\text { elicitation and evolution based on web-enabled collective } \\
\text { intelligence }\end{array}$ & 2013 \\
\hline 41 & Todoran I. et al. & $\begin{array}{l}\text { How cloud providers elicit consumer requirements: An ex- } \\
\text { ploratory study of nineteen companies }\end{array}$ & 2013 \\
\hline 42 & Daramola O. et al. & $\begin{array}{l}\text { A comparative review of } i^{*} \text {-based and use case-based secu- } \\
\text { rity modelling initiatives }\end{array}$ & 2012 \\
\hline 43 & Dias, A.L. et al. & $\begin{array}{l}\text { Increasing the quality of web systems: By inserting require- } \\
\text { ments of accessibility and usability }\end{array}$ & 2012 \\
\hline 44 & Fernandes J. et al. & $\begin{array}{l}\text { IThink: A game-based approach towards improving collabo- } \\
\text { ration and participation in requirement elicitation }\end{array}$ & 2012 \\
\hline 45 & Salini P., Kanmani S. & $\begin{array}{l}\text { Security requirements engineering process for web applica- } \\
\text { tions }\end{array}$ & 2012 \\
\hline 46 & Lim S.L., Finkelstein A. & $\begin{array}{l}\text { Stakerare: Using social networks and collaborative filtering } \\
\text { for large-scale requirements elicitation }\end{array}$ & 2012 \\
\hline 47 & Gonzales C.K., Leroy G. & Eliciting user requirements using Appreciative inquiry & 2011 \\
\hline 48 & Pitula K.,Radhakrishnan T. & $\begin{array}{l}\text { On eliciting requirements from end-users in the ICT4D do- } \\
\text { main }\end{array}$ & 2011 \\
\hline 49 & Mobasher, B. et al. & Recommender systems in requirements engineering & 2011 \\
\hline 50 & Fabian B. et al. & A comparison of security requirements engineering methods & 2010 \\
\hline 51 & Adem, N.A.Z., Kasirun Z.M. & $\begin{array}{l}\text { Automating function points analysis based on functional and } \\
\text { non-functional requirements text }\end{array}$ & 2010 \\
\hline 52 & Smith, C. et al. & $\begin{array}{l}\text { Data structure visualization: The design and implementation } \\
\text { of an animation tool }\end{array}$ & 2010 \\
\hline 53 & $\begin{array}{l}\text { Winschiers-Theophilus } \mathrm{H} \text {. } \\
\text { et al. }\end{array}$ & $\begin{array}{l}\text { Determining requirements within an indigenous knowledge } \\
\text { system of African rural communities }\end{array}$ & 2010 \\
\hline 54 & Seyff N. et al. & Using mobile RE tools to give end-users their own voice & 2010 \\
\hline 55 & Castro-Herrera C. et al. & $\begin{array}{l}\text { A recommender system for requirements elicitation in large- } \\
\text { scale software projects }\end{array}$ & 2009 \\
\hline 56 & Pu Y., Liu Q. & $\begin{array}{l}\text { A viewpoint-oriented requirements elicitation integrated } \\
\text { with aspects }\end{array}$ & 2009 \\
\hline 57 & Sadiq Mohd. et al. & $\begin{array}{l}\text { An approach for eliciting software requirements and its prio- } \\
\text { ritization using analytic hierarchy process }\end{array}$ & 2009 \\
\hline
\end{tabular}




\begin{tabular}{|l|l|l|l|}
\hline$\#$ & Autores & Título & Ano \\
\hline 58 & Guerra-García C. et al. & $\begin{array}{l}\text { DQ-VORD: A methodology for managing and integrating } \\
\text { data quality requirements into software requirement speci- } \\
\text { fication }\end{array}$ & 2009 \\
\hline 59 & Castro-Herrera, C. et al. & $\begin{array}{l}\text { Enhancing stakeholder profiles to improve recommendati- } \\
\text { ons in online requirements elicitation }\end{array}$ & 2009 \\
\hline 60 & Romero M. et al. & $\begin{array}{l}\text { Teaching requirements elicitation within the context of glo- } \\
\text { bal software development }\end{array}$ & 2009 \\
\hline 61 & Motta G. et al. & $\begin{array}{l}\text { User goal-oriented requirements elicitation to improve ac- } \\
\text { ceptance and use: A case study on document management }\end{array}$ & 2009 \\
\hline
\end{tabular}

Quadro 2. Artigos selecionados na RSL. 


\section{REFERÊNCIAS}

Abd-Elmonem, M. A., Nasr, E. S., \& Gheith, M. H. (2017). Automating Requirements Elicitation of Cloud-Based ERPs. In International Conference on Advanced Intelligent Systems and Informatics (p. 171-180). doi: 10.1007/978-3-319-64861$3 \quad 16$

Adem, N. A. Z., \& Kasirun, Z. M. (2010, fev. ). Automating function points analysis based on functional and non functional requirements text. In The 2nd International Conference on Computer and Automation Engineering (ICCAE). IEEE. Recuperado de https://doi.org/10.1109/iccae.2010.5451322 doi: $10.1109 /$ iccae.2010.5451322

Alexa, L., \& Avasilcai, S. (2018). The requirement elicitation process of designing a collaborative environment - the CRE@TIVE.BIZ case. MATEC Web of Conferences, 184, 1-4. Recuperado de https://doi.org/10.1051/matecconf/ 201818404010 doi: $10.1051 /$ matecconf/201818404010

Al-Qudah, D. A., Cristea, A. I., \& Lei, S. (2013). An Exploratory Study to Design an Adaptive Hypermedia system for online-advertisement. In The 9th International Conference on Web Information Systems and Technologies (p. 368-374). Recuperado de http://rgdoi.net/10.13140/ 2.1.3107.9368 doi: 10.13140/2.1.3107.9368

Alvertis, I., Papaspyros, D., Koussouris, S., Mouzakitis, S., \& Askounis, D. (2016). Using Crowdsourced and Anonymized Personas in the Requirements Elicitation and Software Development Phases of Software Engineering. In 11th international conference on availability, reliability and security (ARES). IEEE. Recuperado de https://doi.org/10.1109/ ares.2016.71 doi: 10.1109/ares.2016.71

Angelis, G. D., Ferrari, A., Gnesi, S., \& Polini, A. (2018). Requirements elicitation and refinement in collaborative research projects. Journal of Software: Evolution and Process, 30(12), 1-18. Recuperado de https://doi.org/10.1002/ smr.1990 doi: 10.1002/smr.1990

Araujo, R., Anjos, E., \& Silva, D. R. (2015). Trends in the use of design thinking for embedded systems. In 15th International Conference on Computational Science and Its Applications (p. 82-86). IEEE. Recuperado de https:/ doi.org/10.1109/iccsa.2015.25 doi: 10.1109/iccsa.2015.25

Asghar, A. R., Tabassum, A., Bhatti, S. N., \& Jadi, A. M. (2017). Impact and challenges of requirements elicitation \& prioritization in quality to agile process: Scrum as a case scenario. In International conference on communication technologies (ComTech). IEEE. Recuperado de https://doi.org/10.1109/comtech.2017.8065749 doi: 10.1109/comtech.2017.8065749

Babar, A., Bunker, D., \& Gill, A. Q. (2018). Investigating the Relationship between Business Analysts' Competency and IS Requirements Elicitation: A Thematic-analysis Approach. Communications of the Association for Information Systems, 42(1), 334-362. Recuperado de https://doi.org/ 10.17705/1cais.04212 doi: 10.17705/1cais.04212

Baptista, M. N., \& de Campos, D. C. (2007). Metodologias de pesquisa em ciências: análises quantitativa e qualitativa (2a. ed.). Rio de Janeiro: Livros Técnicos e Científicos.

Batista, E. A. (2003). Uma taxonomia facetada para técnicas de elicitação de requisitos (Dissertação de mestrado, Instituto de Computação, UNICAMP, Campinas, SP, Brasil). Recuperado de http://repositorio.unicamp.br/bitstream/ REPOSIP/276373/1/Batista_EdinelsonAparecido_M.pdf (Dissertação de Mestrado)

Batool, A., Motla, Y. H., Hamid, B., Asghar, S., Riaz, M.,
Mukhtar, M., \& Ahmed, M. (2013). Comparative study of traditional requirement engineering and agile requirement engineering. In 15th International Conference on Advanced Communications Technology (ICACT) (p. 1006-1014).

Buitrón, S. L., Flores-Rios, B. L., \& Pino, F. J. (2018). Elicitación de requisitos no funcionales basada en la gestión de conocimiento de los stakeholders. Ingeniare. Revista chilena de ingeniería, 26 (1), 142-156. doi: 10.4067/S071833052018000100142

Caleb-Solly, P., Dogramadzi, S., Ellender, D., Fear, T., \& Heuvel, H. v. d. (2014). A mixed-method approach to evoke creative and holistic thinking about robots in a home environment. In $A C M / I E E E$ International Conference on Human-Robot Interaction (p. 374-381). ACM Press. doi: $10.1145 / 2559636.2559681$

Cervo, A. L., \& Bervian, P. A. (2007). Metodologia científica: para uso dos estudantes universitários. In Metodologia cientifica: para uso dos estudantes universitários (6a. ed.). S ao Paulo: Pearson Prentice Hall.

Fernandes, J., Duarte, D., Ribeiro, C., Farinha, C., Pereira, J. M., \& da Silva, M. M. (2012). iThink: A game-based approach towards improving collaboration and participation in requirement elicitation. Procedia Computer Science, 15(1), 66-77. doi: 10.1016/j.procs.2012.10.059

Gill, K. D., Zaidi, A. M., \& Kiani, M. M. (2014). Eliciting futuristic end-user requirements through contributory appreciative inquiry. In National software engineering conference (p. 49-54). IEEE. doi: 10.1109/NSEC.2014.6998240

Gonzales, C. K., \& Leroy, G. (2011). Eliciting user requirements using appreciative inquiry. Empirical Software Engineering, 16 (6), 733-772. doi: 10.1007/s10664-011-9156$\mathrm{x}$

Hajri, I., Goknil, A., Briand, L. C., \& Stephany, T. (2018). Configuring use case models in product families. Software Es Systems Modeling, 17(3), 939-971. doi: 10.1007/s10270016-0539-8

Hickey, A. M., \& Davis, A. M. (2003). Elicitation technique selection: how do experts do it? In 11th ieee international requirements engineering conference (p. 169-178). doi: 10.1109/ICRE.2003.1232748

Hidalga, A. N. d. l., Hardisty, A., \& Jones, A. (2016). Scramck: applying a collaborative requirements engineering process for designing a web based e-science toolkit. Requirements Engineering, 21(1), 107-129. doi: 10.1007/s00766014-0212-0

Hosseini, M., Shahri, A., Phalp, K., Taylor, J., Ali, R., \& Dalpiaz, F. (2015). Configuring crowdsourcing for requirements elicitation. In 9th International Conference on Research Challenges in Information Science (RCIS) (p. 133138). doi: 10.1109/RCIS.2015.7128873

Jayatilleke, S., \& Lai, R. (2018). A systematic review of requirements change management. Information and Software Technology, 93, 163-185. doi: 10.1016/j.infsof.2017.09.004

Kiran, H. M., \& Ali, Z. (2018). Requirement elicitation techniques for open source systems: a review. International Journal of Advanced Computer Science and Applications (IJACSA), 9(1), 330-334. doi: 10.14569/IJACSA.2018.090145

Kitchenham, B. (2004). Procedures for performing systematic reviews. Department of Computer Science, Keele University, UK. (Technical Report)

Kitchenham, B., \& Charters, S. (2007). Guidelines for 
performing systematic literature reviews in software engineering. Keele University and Durham University Joint Report. (Technical Report)

Knauss, E., Yussuf, A., Blincoe, K., Damian, D., \& Knauss, A. (2018). Continuous clarification and emergent requirements flows in open-commercial software ecosystems. Requirements Engineering, 23(1), 97-117. doi: 10.1007/s00766016-0259-1

Mishra, D., Aydin, S., Mishra, A., \& Ostrovska, S. (2018). Knowledge management in requirement elicitation: Situational methods view. Computer Standards $\&$ Interfaces, 56(1), 49-61. doi: 10.1016/j.csi.2017.09.004

Mishra, D., Mishra, A., \& Yazici, A. (2008). Successful requirement elicitation by combining requirement engineering techniques. In First international conference on the applications of digital information and web technologies (icadiwt) (p. 258-263). doi: 10.1109/ICADIWT.2008.4664355

Mobasher, B., \& Cleland-Huang, J. (2011). Recommender systems in requirements engineering. Association for the Advancement of Artificial Intelligence (AAAI), 32(3), 81-89. doi: 10.1609/aimag.v32i3.2366

Nuseibeh, B., \& Easterbrook, S. (2000). Requirements engineering: a roadmap. In Proceedings of the conference on the future of software engineering (p. 35-46). doi: $10.1145 / 336512.336523$

Odusote, B., Daramola, O., \& Adigun, M. (2018). Towards an extended misuse case framework for elicitation of cloud dependability requirements. In Saicsit '18 proceedings of the annual conference of the south african institute of computer scientists and information technologists (p. 135-144). doi: $10.1145 / 3278681.3278698$

Oriol, M., Stade, M., Fotrousi, F., Nadal, S., Varga, J., Seyff, N., ... Schmidt, O. (2018). Fame: supporting continuous requirements elicitation by combining user feedback and monitoring. In 2018 ieee 26th international requirements engineering conference (re) (p. 217-227). doi: 10.1109/RE.2018.00030

Pitula, K., \& Radhakrishnan, T. (2011). On eliciting requirements from end-users in the ict4d domain. Requirements Engineering, 16(1), 323. doi: 10.1007/s00766-011-0127-y

Ramakrishnan, L., Poon, S., Hendrix, V., Gunter, D., Pastorello, G. Z., \& Agarwal, D. (2014). Experiences with user-centered design for the tigres workflow api. In 2014 IEEE 10th International Conference on e-Science (p. 290297). doi: 10.1109/eScience.2014.56

Ramesh, M. R., \& Reddy, C. S. (2016). A survey on security requirement elicitation methods: classification, merits and demerits. International Journal of Applied Engineering Research, 11(1), 64-70.

Sadiq, M., Ghafir, S., \& Shahid, M. (2009). An approach for eliciting software requirements and its prioritization using analytic hierarchy process. In 2009 international conference on advances in recent technologies in communication and computing (p. 790-795). doi: 10.1109/ARTCom.2009.58

Sheffield, J., \& Lemétayer, J. (2013). Factors associated with the software development agility of successful projects. International Journal of Project Management, 31(1), 459472. doi: 10.1016/j.ijproman.2012.09.011

Smith, C., Strauss, J., \& Maher, P. (2010). Data structure visualization: the design and implementation of an animation tool. In Proceedings of the 48th annual southeast regional conference (p. 1-6). doi: 10.1145/1900008.1900105
Thomas, K., Bandara, A. K., Price, B. A., \& Nuseibeh, B. (2014). Distilling privacy requirements for mobile applications. In Proceedings of the 36th international conference on software engineering (p. 871-882). doi: $10.1145 / 2568225.2568240$

Vijayan, J., Raju, G., \& Joseph, M. (2016). Collaborative requirements elicitation using elicitation tool for small projects. In 2016 international conference on signal processing, communication, power and embedded system (scopes) ( $\mathrm{p}$. 340-344). doi: 10.1145/10.1109/SCOPES.2016.7955848

Wertsch, J. V. (1981). The concept of activity in soviet psychology: an introduction. In J. V. Wertsch (Ed.), The concept of activity in soviet psychology (p. 86-99). Armonk: M. E. Sharpe.

Younas, M., Jawawi, D., Ghani, I., \& Kazmi, R. (2017). Non-functional requirements elicitation guideline for agile methods. Journal of Telecommunication, Electronic and Computer Engineering, 9(1), 137-142.
Como citar este artigo (APA):

Alflen, N. C. \& Prado, E. P. V. (2021). Técnicas de elicitação de requisitos no desenvolvimento de software: uma revisão sistemática da literatura. AtoZ: novas práticas em informação e conhecimento, 10(1), 39 - 49. Recuperado de: http://dx.doi.org/10.5380/atoz.v10i1 .77393 